\title{
Turbina eólica para agricultura familiar do semiárido com inovações tecnológicas para baixas velocidades de vento ${ }^{1}$
}

\author{
Wind turbine for family farming in semi-arid areas with technological innovations for \\ low wind speeds
}

\author{
Daniel Albiero $^{2 *}$, Sérgio Daher ${ }^{3}$, Leonardo de Almeida Monteiro e Francisco José Firmino Canafístula $^{5}$
}

\begin{abstract}
RESUMO - A agricultura familiar é um segmento importante do agronegócio brasileiro e tem grande participação na produção de alimentos essenciais para a segurança alimentar do Brasil, por isso, é preciso o fornecimento de energia para esses agricultores. Uma alternativa é a energia eólica, no entanto um desafio para usar o vento em pequenas propriedades no Estado do Ceará é a condição operacional do vento que é de baixa velocidade (ventos $<6 \mathrm{~m} \mathrm{~s}^{-1}$ ). $\mathrm{O}$ objetivo deste trabalho foi propor um sistema eólico para pequenas propriedades do Estado do Ceará com inovações tecnológicas. Todos os sistemas foram concebidos e dimensionados em uma turbina inovadora e apropriada para as pequenas propriedades. A estimativa de custo indica um baixo valor de aquisição e através de redes inteligentes e da geração distribuída pode-se gerar renda.
\end{abstract}

Palavras-chave: Energia Eólica. Energia-Fontes alternativas. Energia renovável.

\begin{abstract}
Family farming is an important segment of Brazilian agribusiness, and has a large part in producing that food which is essential for food security in Brazil, so the supply of energy to these farmers becomes necessary. One alternative is wind energy; however a challenge in using the wind on small properties in the State of Ceará are its lowspeed conditions of operation (winds $<6 \mathrm{~m} \mathrm{~s}-1$ ). The objective of this work was to propose a wind-energy system for small farms in the State of Ceará, having technological innovations. All the systems were designed and scaled employing an innovative turbine, and are suitable for small properties. The cost estimate indicates a low value for acquisition, and by using smart grids and the distribution of generated energy, income can be produced.
\end{abstract}

Key words: Wind Energy. Operating conditions. Renewable energy.

\footnotetext{
*Autor para correspondência

${ }^{1}$ Recebido para publicação 16/03/2012; aprovado em 14/09/2013

Pesquisa financiada pelo CNPq através de Bolsa de Produtividade em Desenvolvimento Tecnológico e Extensão Inovadora

${ }^{2}$ Departamento de Engenharia Agrícola/DENA/CCA/UFC, Campus do Pici, Bloco 804, Fortaleza-CE, Brasil, 60.455-760, dalbiero@ufc.br ${ }^{3}$ Departamento de Engenharia Elétrica/DEE/CT, Campus do Pici, Bloco 710, Fortaleza-CE, Brasil, 60.455-760, sdaher@ dee.ufc.br

${ }_{4}^{4}$ Departamento de Engenharia Agrícola/DENA/CCA/UFC, Campus do Pici, Bloco 804, Fortaleza-CE, Brasil, 60.455-760, aiveca@ ufc.br

${ }_{5}^{5}$ Departamento de Engenharia Agrícola/DENA/CCA/UFC, Campus do Pici, Bloco 804, Fortaleza-CE, Brasil, 60.455-760, firmino@ufc.br
} 


\section{INTRODUÇÃO}

A agricultura familiar é um setor muito importante para o agronegócio brasileiro, pois detém a maior parte da produção de diversos gêneros alimentícios essenciais para a segurança alimentar da nação. Embora o programa Luz para Todos (BRASIL, 2011) tenha tornado acessível a energia elétrica para a maioria das propriedades familiares do Ceará, o custo desta energia é alto para o poder aquisitivo e produtivo deste setor agrícola. Portanto, uma forma de melhorar a renda da agricultura familiar é possibilitar o acesso à tecnologia de aerogeradores que devem ser apropriados para cada cenário (BARIN et al., 2010; WANG et al., 2008), pois Ávila et al. (2006) afirmam que a geração eólica não contamina o ar, solo e água e Rocha et al. (2010) ainda pontua que a mesma é inesgotável.

No entanto existe um grande desafio para a efetivação da energia eólica para a agricultura familiar no Ceará: a adequação às condições operacionais de baixo vento (ventos $<6 \mathrm{~m} \mathrm{~s}^{-1}$ ). Se não bastasse a natureza estocástica do vento que é altamente errático segundo Gonzalez et al. (2012), Maria (2008) esclarecem que o regime do fluxo de ar sobre um aerofólio é caracterizado por pequeno número de Reynolds nas regiões aonde a agricultura familiar se desenvolve, da ordem de $10^{4}$ a $10^{5}$, o que difere significativamente das condições normais de operação dos aerofólios próprios para aerogeradores que são ensaiados em números de Reynolds da ordem de $10^{5}$ a $10^{6}$.

Neste contexto, inovações tecnológicas devem ser utilizadas para superar tal dificuldade. Estudos de Carmichael (1982) identificaram o aerofólio Eppler 61 (E61) próprio para aplicações em planadores como detentor de excelente desempenho a baixas velocidades de vento e Habash et al. (2011) afirmam que um sistema de rotores contra-rotativos gera $60 \%$ mais energia do que em um sistema mono-rotor em função do reaproveitamento da energia restante na esteira de ar segundo Sorensen et al. (2008).

Nho et al. (2012) provaram que os vórtices de ponta de asa aumentam as perdas aerodinâmicas, assim se faz necessário o uso de winglets para melhora o desempenho aerodinâmico dos aerofólios. Whitcomb (1976) sugeriu a adoção de winglets e neste contexto de aumento de eficiência uma inovação interessante é a tecnologia TARP (Toroidal Accelerator Rotor Plataform) (WEISBRICH; RAINEY, 1977) que aumenta a velocidade do vento em $180 \%$ através de uma forma geométrica toroidal que direciona o vento, amplificando-o com o efeito venturi.

Um componente de valor expressivo em sistemas eólicos que deve ser considerado é o gerador elétrico de imãs permanentes (PINILLA; MARTINEZ, 2012; RAMIREZ et al., 2012), com grande número de pólos (BYSTRIYK; SULLIVAN, 2011), segundo Daher (1997) é viável economicamente e tecnicamente a geração de energia elétrica através da adaptação de um motor de indução trifásico (MIT) com auto-excitação através de um inversor estático; segundo Daher (1997) este dimensionamento permite o acoplamento mecânico direto entre gerador e rotor o que torna o sistema mais simples, robusto e barato. Embora não tenha alta eficiência, apresenta inegáveis vantagens em relação ao custo de aquisição e manutenção além de fácil acesso em qualquer localidade nacional.

Outra inovação necessária segundo Vogt (2010) é a utilização da metodologia definida por Hansen (2004) que desenvolveu a estrutura de rotores eólicos com fibras. No entanto, Dicorato et al. (2012) alertam que estas tecnologias devem se preocupar com o comportamento do gerador sob diferentes regimes de distúrbios, principalmente considerando as novas características operacionais que se perfazem com a adoção destas novas tecnologias.

O objetivo deste trabalho foi propor um sistema para fornecer energia elétrica através do vento para uma propriedade familiar no Estado do Ceará com as seguintes inovações tecnológicas: aerofólio inovador para aplicações de geração eólica; rotores contra-rotativos; materiais em fibra vegetal; gerador de indução através da adaptação de um motor de indução trifásico; ponta das pás dos rotores com winglets e sistema toroidal de aumento de velocidade de vento-TARP.

\section{MATERIAL E MÉTODOS}

A Carga elétrica a ser fornecida se refere a: bomba de irrigação Schneider de $368 \mathrm{~W}$; computador: $180 \mathrm{~W}$; geladeira: $130 \mathrm{~W}$; televisão: $110 \mathrm{~W}$. Total: $970 \mathrm{~W}$.

Segundo Daher (1997), é viável econômico e tecnicamente a geração de energia elétrica através da adaptação de um motor de indução trifásico de $7,5 \mathrm{~kW}$. Para que tal motor gere uma potência de saída de 970 W é necessária uma rotação do rotor de $47,1 \mathrm{rad} \mathrm{s}^{-1}(450$ RPM), eficiência elétrica de $70 \%$, escorregamento de $8 \%$, amperagem de 10,4 A e voltagem do enrolamento de $40 \mathrm{~V}$. Todos estes parâmetros foram determinados teoricamente e experimentalmente por Daher (1997). A potência Gerada de Saída é dada pela equação 1:

$$
-P g=P m+P d
$$

onde: Pg é a potência gerada de saída (W); Pm é a potência Mecânica (W); Pd é a potência Dissipada (W). Segundo Daher (1997) a Potência Dissipada é dada pela equação 2 : 


$$
P d=\left[\left(i_{1}^{2} \cdot R_{1}\right)+\left(i_{2}^{2} \cdot R_{2}\right)+\left(\frac{V m^{2}}{R c}\right)\right]
$$

onde: $\mathrm{i}_{1}$ é a corrente no enrolamento do estator (A); $\mathrm{i}_{2}$ é a corrente no rotor (A); $R_{1}$ é a resistência do enrolamento do estator $(\Omega) ; \mathrm{R}_{2}$ é a resistência do rotor $(\Omega) ; R c$ é a resistência equivalente das perdas no ferro $(\Omega) ; V m$ é a tensão sob a indução de magnetização (V). Segundo Daher (1997) a tensão de indução é dada pela equação 3:

$$
|V m|=\left|i_{2}\right| \cdot \sqrt{x_{2}^{2}+\left(\frac{R_{2}}{s}\right)^{2}}
$$

onde: $\mathrm{s}$ é o escorregamento do motor; $\mathrm{x}_{2}$ é a reatância do rotor $(\Omega)$ e é dada pela equação 4 :

$$
\mathrm{x}_{2}=2 . \pi \cdot \mathrm{L}_{2} \cdot \mathrm{f}
$$

onde: $L_{2}$ é a indutância de dispersão do rotor $(\Omega)$; f é a frequiência nominal da máquina $(\mathrm{Hz})$.

Para tal motor, Daher (1997) obteve os seguintes dados experimentais: $\mathrm{i}_{1}=10,4 \mathrm{~A} ; \mathrm{i}_{2}=10,4 \mathrm{~A} ; \mathrm{R}_{1}=0,65 \Omega$; $\mathrm{R}_{2}=0,99 \Omega ; \mathrm{s}=0,08 ; \mathrm{Rc}=143,4 \Omega ; \mathrm{L}_{2}=0,00452 \Omega$; $\mathrm{f}=60 \mathrm{~Hz}$. Com estes dados foi calculado $\mathrm{Vm}=129 \mathrm{~V}$, e posteriormente foi calculada a Potência dissipada: $P d=293,4$ W. Portanto, a Potência mecânica necessária para gerar $P g=970 \mathrm{~W}$ é de $P m=1263 \mathrm{~W}$. No entanto, Fitzgerald et al. (2006) estabelece que a potência no eixo do gerador (Peixo) deve ser a potência mecânica $(\mathrm{Pm})$ mais as perdas mecânicas definida como o dispêndio de potência referido à movimentação do ventilador do motor e as perdas de atrito dos rolamentos (Ppmec). Estas perdas são padronizadas como $10 \%$ do valor da potência no eixo, assim, potência no eixo é:

Peixo $=\mathrm{Pm}+$ Ppmec $\rightarrow$ Peixo $=\mathrm{Pm}+(0,1 \cdot$ Peixo $)$
Portanto, a potência necessária no eixo do gerador para gerar $970 \mathrm{~W}$ é de 1403,3 W.

De posse da potência necessária no eixo do gerador é possível dimensionar o sistema de rotor para captar tal potência do vento. Em função da velocidade de vento baixa das regiões onde as propriedades familiares se localizam, foi considerada uma velocidade para produção em regime de $3,45 \mathrm{~m} \mathrm{~s}^{-1}$.

Para otimizar a captação da energia cinética do vento nestas condições de baixo vento, foi selecionado o aerofólio E61, próprio para uso em planadores, sua caracterização é dada por Airfoil Investigation Database. (2011) na Figura 1 e Tabela 1.

Este aerofólio possibilita ótima eficiência aerodinâmica para as condições de vento de $3,45 \mathrm{~m} \mathrm{~s}^{-1}$, no entanto somente tal característica não é suficiente para fornecer a potência solicitada pelo gerador. Para melhorar as características do E61 foi adotada a

Tabela 1 - Parâmetros do Aerofólio E61 para Reynolds de 75000 (AIRFOIL INVESTIGATION DATABASE., 2011)

\begin{tabular}{ll}
\hline \multicolumn{1}{c}{ Parâmetro } & Valor \\
\hline Espessura & $5,7 \%$ \\
Camber & $6,7 \%$ \\
Coeficiente de Sustentação Máximo & 1,6 \\
Coefic. de Arrasto para Sustentação Máxima & 0,022 \\
Ângulo de ataque para sustentação Máxima & $5^{\circ}$ \\
Ângulo de Stall & $5^{\circ}$ \\
Máximo Razão L/D & 80 \\
Ângulo para Máxima Razão L/D & $3,5^{\circ}$ \\
Ângulo para sustentação Zero & $-9^{\circ}$ \\
\hline
\end{tabular}

Figura 1 - Perfil do Aerofólio E61 (AIRFOIL INVESTIGATION DATABASE, 2011)

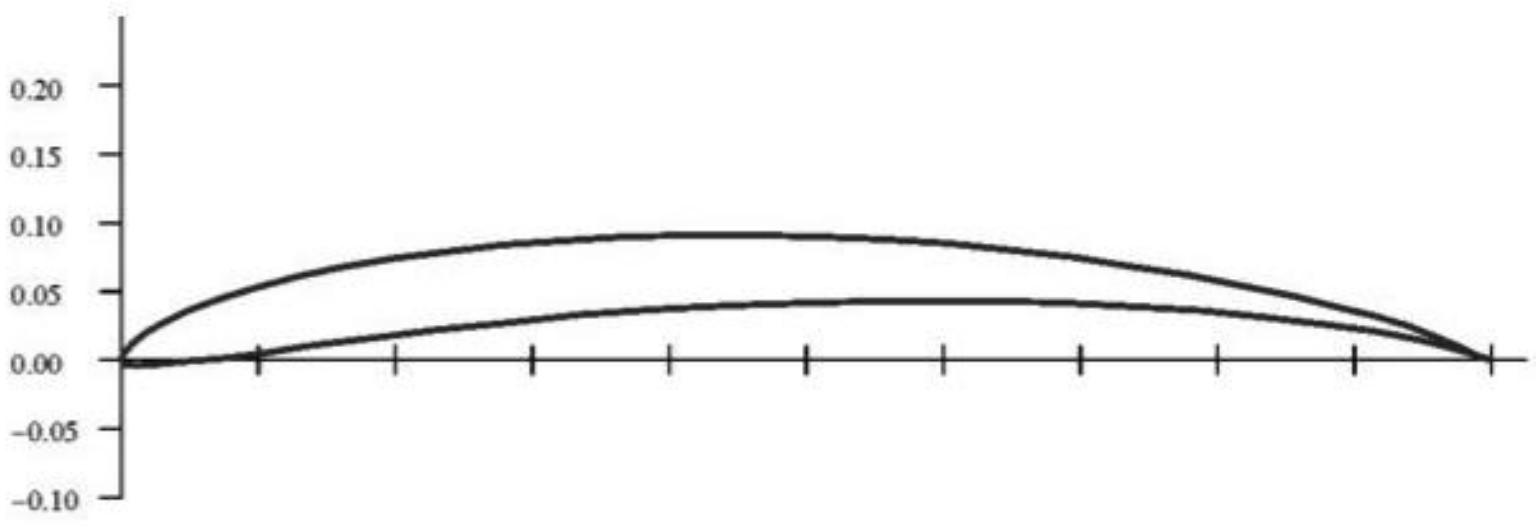


tecnologia de Winglets, que são pequenas asas dispostas nas pontas das pás do rotor. Assim, neste trabalho serão considerados o Coeficiente de Sustentação Máximo para o aerofólio E61 com winglet de $\mathrm{Cl}=1,92$ e o Coeficiente de Arrasto para Sustentação Máxima de $\mathrm{Cd}=0,02$. O ângulo de ataque $(\alpha)$ será considerado de $4^{\circ}$. Deve ser ressaltado que devido à geometria supercrítica e fina deste aerofólio, sua aplicação em turbinas eólicas fica restrita a rotores de até 2,5 metros de raio.

As dimensões das pás do aerogerador foram calculadas utilizando a teoria do BEM - Blade Element Momentum Theory, Teoria do Momento do Elemento de Pá (MANWELL; MCGOWAN; ROGERS, 2002).

Segundo Manwell, McGowan e Rogers (2002) a potência extraível da energia cinética do vento é dada pela de acordo com a equação 6 :

Pot $_{\text {eixo }}=(1 / 2) C p \cdot \rho \cdot V_{\infty}^{3} \cdot \pi \cdot R^{2}$

onde: $C p$ é o coeficiente de potência; $\rho$ - Densidade do ar $\left(\mathrm{kg} \mathrm{m}^{-3}\right) \mathrm{V}_{\infty}$ - Velocidade do vento $\left(\mathrm{m} \mathrm{s}^{-1}\right) ; R$ - Raio do rotor $(\mathrm{m})$.

Segundo Manwell, McGowan e Rogers (2002) o coeficiente de potência é dado pela equação 7:

$$
C p=\frac{8}{\lambda} \int_{\lambda r}^{\lambda} F \cdot a \cdot(1-a) \cdot\left[1-\left(\frac{C d}{C l}\right) \cdot \cot (\beta)\right] \cdot \lambda r^{3} \cdot d \lambda r
$$

onde: $\lambda$ é a velocidade específica da ponta da pá e $\lambda \mathrm{r}$ é a velocidade específica parcial, segundo Manwell, McGowan e Rogers (2002) é dado pela equação 8:

$$
\lambda=\frac{\omega \cdot R}{V_{\infty}}
$$

onde: $\omega$ é a rotação do rotor $\left(\operatorname{rad}_{.} \mathrm{s}^{-1}\right)$; a é o fator de indução axial dado pela equação 9 Manwell, McGowan e Rogers (2002).

$a=1 /\left[1+4 \sin ^{2} \varphi / \sigma^{\prime} C_{I} \cos \sigma\right.$

onde: $\varphi$ é o ângulo de vento relativo (radianos) dado segundo Manwell, McGowan e Rogers (2002) por $\varphi$ $=2 /(3 . \lambda r) ; \sigma$ ' é a solidez do rotor, segundo Manwell, McGowan e Rogers (2002) dado pela equação 10:

$\sigma^{\prime}=\frac{N \cdot c}{2 \cdot \pi \cdot r}$

onde: $N$ é o número de pás do rotor; $\mathrm{r}$ é o raio parcial do rotor (m); c é a corda do aerofólio parcial da pá do rotor dada segundo Manwell, McGowan e Rogers (2002) pela expressão 11:

$$
c=\frac{8 \cdot \pi \cdot r \cdot \operatorname{sen}(\varphi)}{3 \cdot N \cdot C l \cdot \lambda r}
$$

onde: a' é o fator de indução angular segundo Manwell, McGowan e Rogers (2002) dado pela equação 12.

$a ́=1 /\left[\left(4 \cos \varphi /\left(\sigma^{\prime} C_{I}\right)\right)-1\right]$

onde: $\beta$ é o ângulo de pitch, (radianos), sabendo que $\beta=$ $\varphi-\alpha ; F$ é fator de correção devido a perdas na ponta e na raiz da pá, segundo Manwell, McGowan e Rogers (2002): $\mathrm{F}=\mathrm{f}(\mu) . \mathrm{fr}(\mu) ; \mathrm{f}(\mu)$ é o fator de correção devido a perdas na ponta da pá e é dado pela equação 13 :

$$
f(\mu)=\frac{2}{\pi} \cos \left[e^{((N / 2)(1-\mu) / \mu) \sqrt{1+(\lambda \mu)^{2} /(1-a)^{2}}}\right]
$$

onde: $\mu$ é a razão parcial do raio do rotor, dado por $\mu=r / R$ (Equação 14);

$$
f_{R}(\mu)=\frac{2}{\mu} \cos \left[e^{-N / 2\left(\mu-\mu_{R} / \mu\right) \sqrt{1+(\lambda \mu)^{2} /(1-a)^{2}}}\right]
$$

O ângulo de torção do aerofólio $(\theta)$ segundo Manwell, McGowan e Rogers (2002) é função da relação entre o ângulo de pitch parcial ( $\beta$ r) e o ângulo de pitch da ponta da pá $\beta \mathrm{t}: \theta=\beta \mathrm{r} / \beta \mathrm{t}$ (radianos).

Para suprir a potência necessária ao gerador à velocidade de vento de $3,45 \mathrm{~m} \mathrm{~s}^{-1}$ com densidade de $1,23 \mathrm{~kg} \mathrm{~m}^{-3}\left(30{ }^{\circ} \mathrm{C}\right.$ ) é preciso desenvolver um rotor com diâmetro muito grande, cálculos preliminares prescrevem um raio do rotor com o aerofólio E61 e winglets de 9 metros.

Esta dimensão impossibilita qualquer tentativa de adequar este sistema às condições técnicas e econômicas da agricultura familiar. Portanto, foi adotada a tecnologia TARP (Toroidal Accelerator Rotor Plataform) (WEISBRICH; RAINEY, 1977) que aumenta a velocidade do vento em $180 \%$. Considerando um vento de $3,45 \mathrm{~m} \mathrm{~s}^{-1}$ com a tecnologia TARP o vento alcança $6,2 \mathrm{~m} \mathrm{~s}^{-1}$.

Nestas condições operacionais considerando uma rotação de projeto de $9,42 \mathrm{rad} \mathrm{s}^{-1}$ (90 RPM), configuração de 6 pás e raio do rotor de 1,8 metros foi dimensionada a pá, Figura $2 \mathrm{a}$, e o winglet adjacente, Figura $2 \mathrm{~b}$. Esta configuração teve $C p$ calculado de 0,31 e $\lambda$ de 2,73. $\mathrm{O}$ winglet foi projetado seguindo recomendações de George (2011) que afirmou que o melhor desempenho foi obtido com winglets que são continuação da raiz da pá (blended), as dimensões seguiram recomendações de Johansen e Sorensen (2007) que determinaram a melhor configuração como aquela em que a altura do winglet 
tem $4 \%$ do raio da pá, raio de curvatura de $12,5 \%$ da altura do winglet, ângulo de torção de $4^{\circ}$ e enflechamento de $0^{\circ}$, Figura 2b. A estrutura da pá será construída seguindo recomendações de Vogt (2010) utilizando como matriz as fibras vegetais de carnaúba e resina de mamona. O autor supracitado, em experimentos, determinou que pás de rotores eólicos fabricados com fibras vegetais suportam ventos de rajadas acima de $42 \mathrm{~m} \mathrm{~s}^{-1}\left(150 \mathrm{~km} \cdot \mathrm{h}^{-1}\right)$, sendo viáveis tecnicamente em função das características mecânicas do material biológico. Estudos de vida útil de materiais compósitos com fibras vegetais demonstraram que tais materiais podem facilmente ultrapassar 10 anos de duração (FREIRE; BERALDO, 2003).

Nesta configuração é possível gerar 518 W. Habash et al. (2011) afirmam que um sistema de rotores contrarotativos gera $60 \%$ mais energia do que em um sistema mono-rotor. Portanto, a energia gerada pelo sistema na configuração contra-rotativa será de 829 W. Em função da tecnologia TARP, a melhor configuração é constituída por dois grupos de rotores contra-rotativos que devem se localizar nas alas do Toróide, Figura 3. A carenagem do sistema TARPfoi projetada para ser coberta por fibras vegetais de carnaúba que além de possibilitarem uma superfície lisa ao fluxo de ar têm a vantagem de ser um material de fácil acesso e sustentável ambientalmente. Portanto, se considerarmos dois rotores acionando um gerador através de sistema de polias e correias (eficiência mecânica de 97\%) tem-se 1.657 W, o que é suficiente para acionar o gerador de indução trifásico descrito por Daher (1997).

O sistema contra-rotativo foi projetado em função do torque e rotação necessários ao gerador (47,1 $\mathrm{rad} \mathrm{s}^{-1}$ x 29,8 N.m). Sabendo que a rotação do rotor $\left(9,42 \mathrm{rad} \mathrm{s}^{-1}\right)$ deve ser multiplicada por 5 para atingir a rotação de 47,1 $\mathrm{rad} \mathrm{s}^{-1}$, foi projetado um sistema de polias com relação de transmissão 1: 5 e eficiência de $97 \%$, nesta configuração cada conjunto contra-rotativo deve fornecer 74,5 $\mathrm{N} \mathrm{m}^{-1}$ a 9,42 $\mathrm{rad} \mathrm{s}^{-1}$, totalizando $149 \mathrm{~N} . \mathrm{m}$ que se converte em $29,8 \mathrm{~N} \mathrm{~m}^{-1}$ a $47,1 \mathrm{rad} . \mathrm{s}^{-1}$. Considerando a potência fornecida por um conjunto contra-rotativo de 829 W e a rotação de $90 \mathrm{rpm}$, tem-se um torque de 80 N.m que é suficiente para suprir a necessidade de torque.

Nestas condições, o conjunto contra-rotativo é sincronizado por um sistema de diferencial composto por 4 engrenagens satélites HKH modelo SM4-25 e respectivos rolamentos cônicos para suporte dos cubos dos rotores, eixo e engrenagens (Figura 4).

Para controle do passo do rotor foi projetado um mecanismo acionado por força centrífuga baseado em Park (1981). O cálculo da massa de acionamento e a dimensão do braço de acionamento seguiram recomendações de Collins (2006). A velocidade do vento limite de operação

Figura 2 - (a) Plano de seções da pá do rotor (mm); (b) Geometria do winglet (mm)

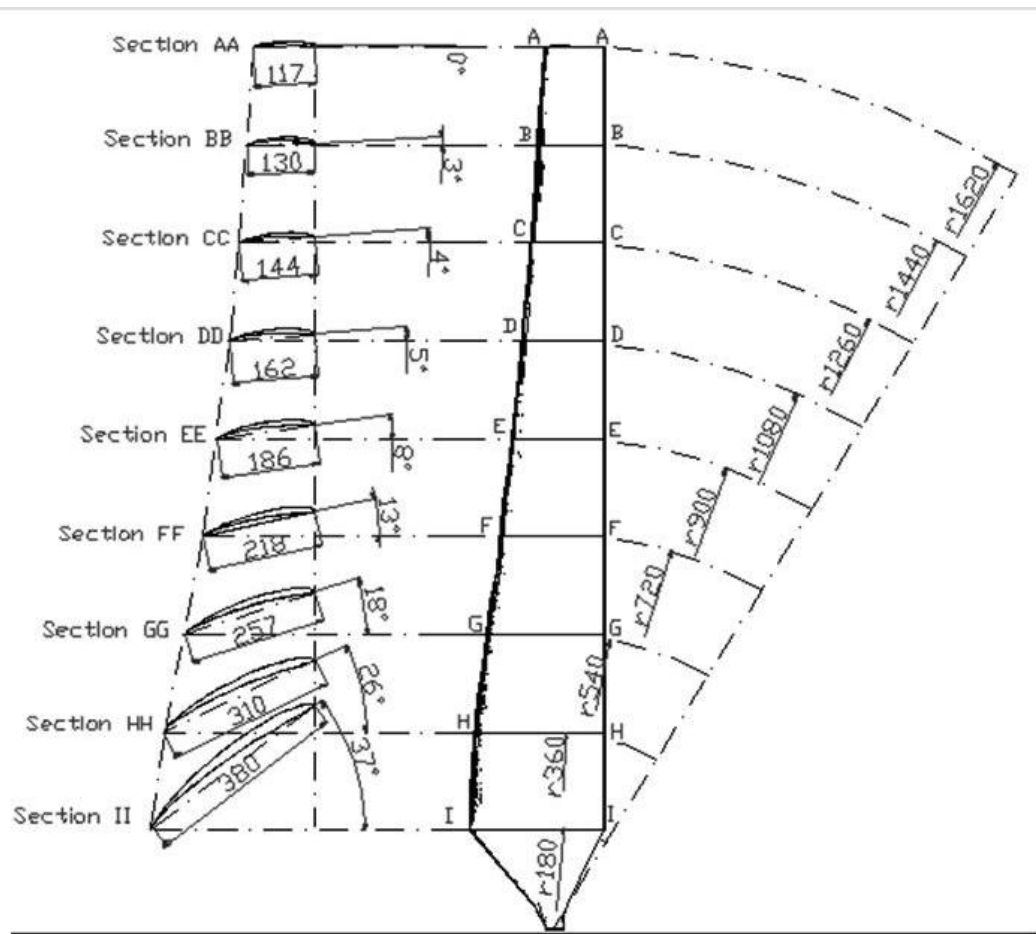

(a)

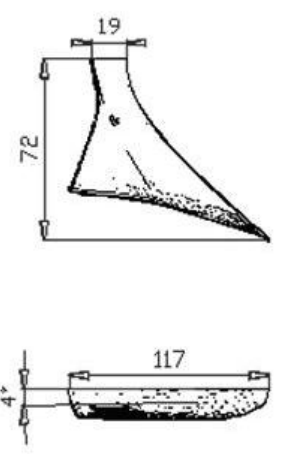

(b) 
foi especificada com $20 \mathrm{~m} \mathrm{~s}^{-1}$ o que em função da velocidade específica da ponta da pá $(\lambda=2,73)$ representa uma rotação de corte de $28 \mathrm{rad} \mathrm{s}^{-1}$ (267 RPM).

Para que o mecanismo apresentado na Figura 5 atue de forma a embandeirar (direcionar as pás a ficarem na mesma direção do fluxo de vento) o rotor à rotação de corte é preciso uma massa para cada pá de $0,85 \mathrm{~kg}$ acoplada a um braço de $0,15 \mathrm{~m}$ de comprimento localizado a $0,15 \mathrm{~m}$ do centro do rotor. Para contraposição a ação das massas foi escolhida uma mola helicoidal de compressão com constante de mola de $1.000 \mathrm{~N} \mathrm{~m}^{-1}$, esta mola está localizada

Figura 3 - (a) Disposição dos rotores no TARP; (b) Vista em perspectiva do TARP

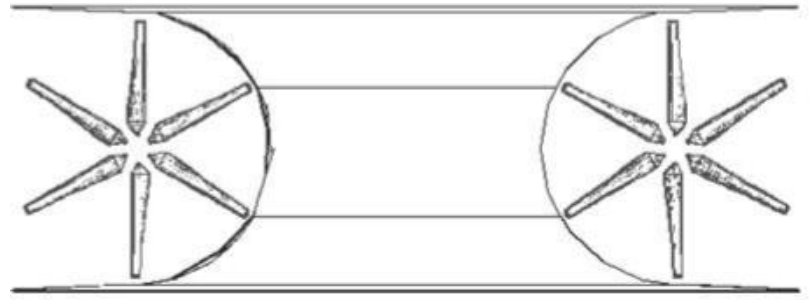

(a)

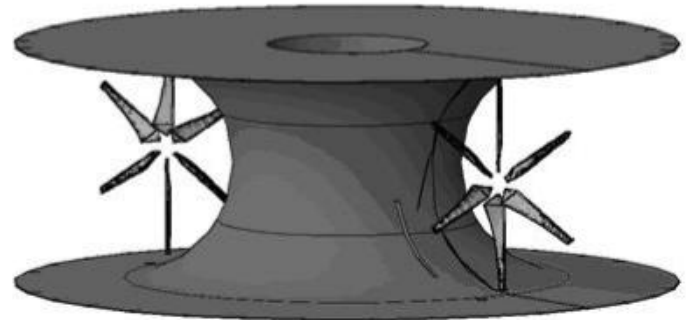

(b)

Figura 4 - (a) Desenho esquemático do diferencial contra-rotativo; (b) Vista em perspectiva do mecanismo diferencial contra-rotativo

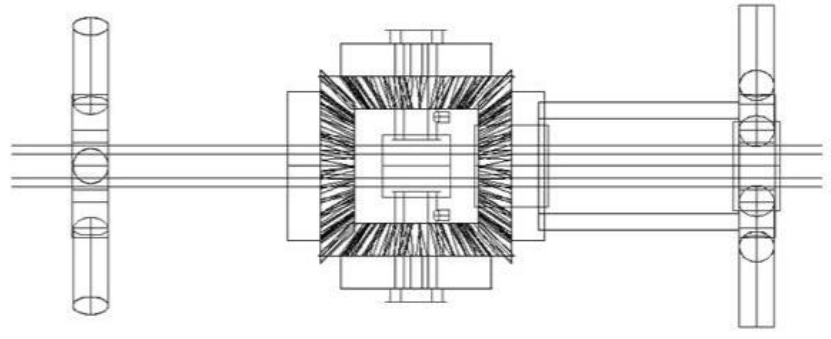

(a)

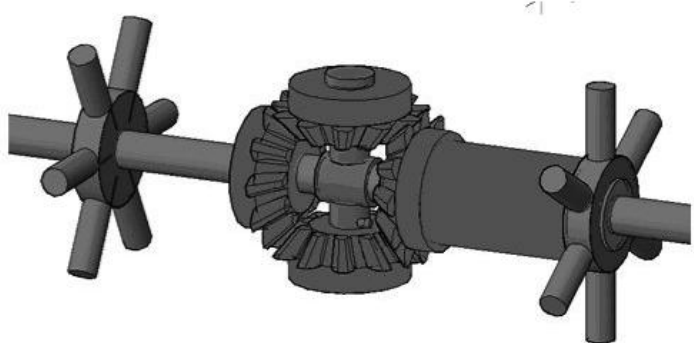

(b)

Figura 5 - Sistema de controle de passo: (a) desenho esquemático; (b) vista renderizada; (c) vista perspectiva dos mecanismos de controle de passo do rotor principal (frontal) e do contra-rotor (traseiro), notar o diferencial contra-rotativo no centro do sistema

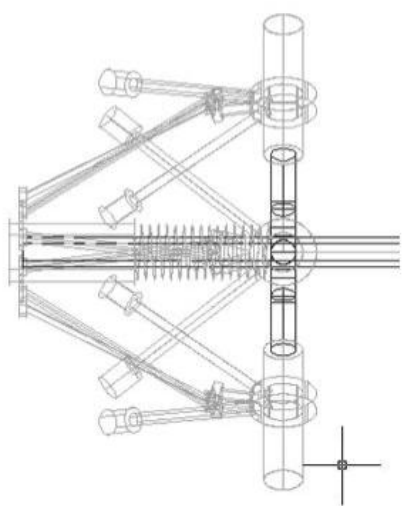

(a)

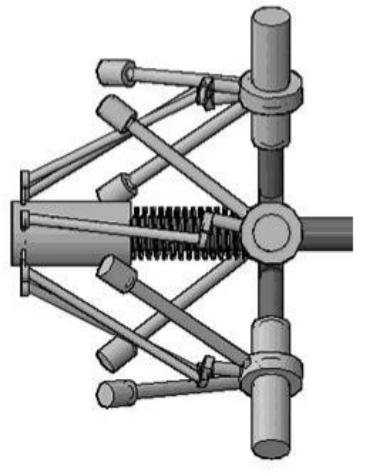

(b)

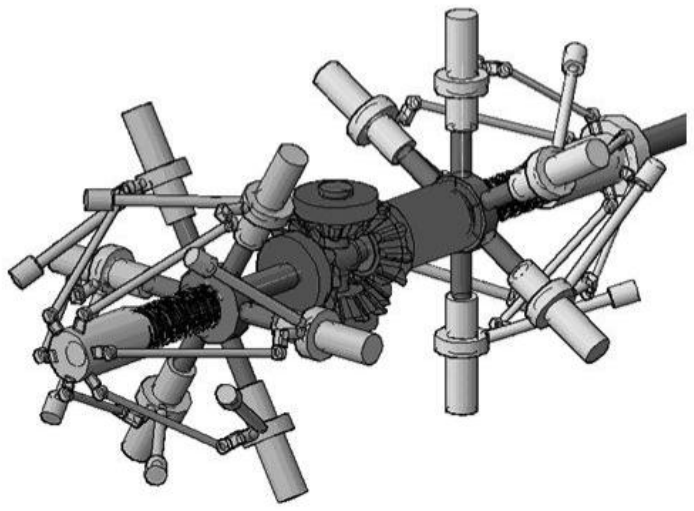

(c) 
no pivô central dos braços. Foi escolhido o material chumbo para compor as massas $\left(\rho=11.150 \mathrm{~kg} \mathrm{~m}^{-3}\right)$, que determina uma geometria das massas cilíndrica com raio de $0,01 \mathrm{~m}$ e altura de $0,0254 \mathrm{~m}$.

A configuração dos rotores contra-rotativos e dos mecanismos associados (diferencial e controle de passo) conduziu a uma geometria estrutural em balanço do eixo principal. Para o cálculo do diâmetro do eixo, seguiuse metodologia definida por Collins (2006) baseada na prevenção de falhas, principalmente em relação aos efeitos de fadiga do material, assim o diâmetro do eixo é calculado pela seguinte equação 15 :

$$
d=\sqrt[3]{\frac{32 \cdot n d}{\pi \cdot 0,5 \cdot S u} \cdot \sqrt{(k f . M a)^{2}+\left(0,75 \cdot T^{2}\right)}}
$$

onde: $n d$ é o fator de segurança; $S u$ é a tensão de resistência a ruptura do aço $(\mathrm{Pa}) ; k f$ o fator de correção para flexão variável; $M a$ é o momento fletor variável (N.m); $T$ é o torque aplicado ao eixo (N.m).

Foi feita uma estimativa dos pesos dos componentes em balanço sobre o eixo: mecanismo de passo frontal $(\mathrm{P} 1)=67,5 \mathrm{~N}$; rotor + cubo frontal $(\mathrm{P} 2)=$ $24 \mathrm{~N}$; diferencial contra-rotativo $(\mathrm{P} 3)=71 \mathrm{~N}$; mecanismo de passo traseiro $(\mathrm{P} 4)=67,5 \mathrm{~N}$; rotor + cubo traseiro $(\mathrm{P} 5)=24 \mathrm{~N}$. As distâncias entre os elementos em balanço e o rolamento são apresentadas na Figura 6.

Figura 6 - Diagrama do eixo principal (mm)

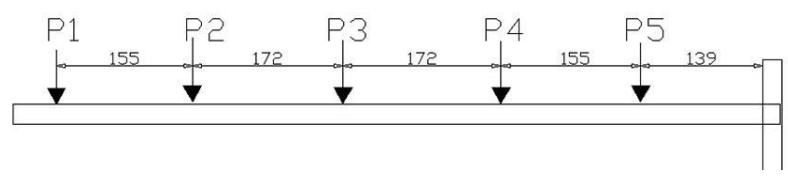

Para aço $1045 \mathrm{CD}$ com $630 \mathrm{MPa}$ de resistência a ruptura (COLLINS, 2006), fator de segurança 2, fator de correção 1,6, Torque no eixo de 80 N.m, e respectivos momentos fletores para cada elemento, tem-se um diâmetro para a menor seção (P1-P2) de 0,0185 m e um diâmetro para a maior seção (P5-rolamento) de 0,022 m. Para efeito de simplificação na contrução mecânica escolheu-se o diâmetro de 0,0254 m para todo o eixo.

Para melhor eficiência aerodinâmica do conjunto de rotores foi projetado um Hub para conduzir o fluxo de ar na região central do rotor para a raiz da pá e evitar turbulências provenientes dos mecanismos. Para a determinação da geometria e dimensão do Hub foi seguida metodologia de Eck (1973) que prescreve a determinação do raio do Hub em função da relação entre a velocidade meridional na raiz das pás e a velocidade periférica do rotor $(\mathrm{cm} / \mathrm{cu})$ em conjunto com o gráfico característico de Hub (ECK, 1973).

\section{RESULTADOS E DISCUSSÃO}

Na figura 7 é possível vislumbrar o aspecto geral do sistema eólico projetado. O diâmetro do sistema TARP é de 10 metros. A altura em relação ao solo do centro dos rotores é de 2,5 metros.

Figura 7- Vista geral em perspectiva do sistema eólico proposto

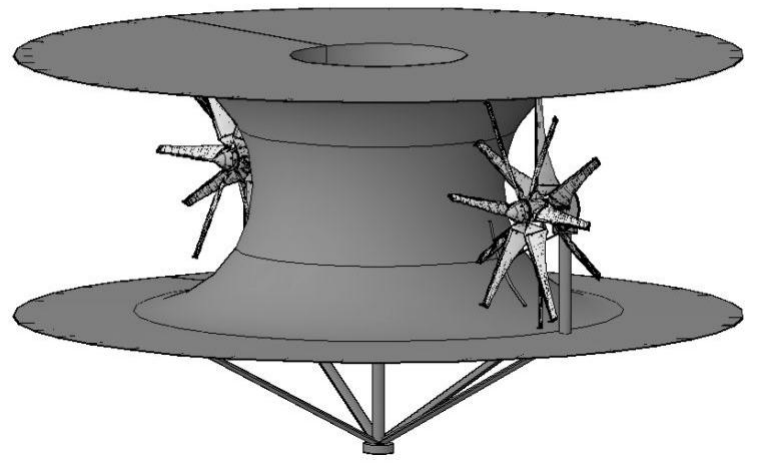

A base móvel e as torres de suporte dos rotores são de tubos de aço 1020 (Figura 8). Os rotores são fixos na torre de suporte e a orientação do sistema em relação ao vento se dá segundo Weisbrich a Rainey (1977) em função da resistência ao arrasto dos conjuntos de rotores que devido à configuração simétrica faz com que a resultante de forças rotacione a base móvel orientando-a sempre na direção ortogonal ao vento.

O sistema de suporte dos rotores, a base móvel, os rotores e respectivos mecanismos foram dimensionados para suportarem ventos de até $30 \mathrm{~m} \mathrm{~s}^{-1}$. Lembrando que muito antes desta velocidade os rotores, devido aos sistemas de controle de passo, estarão embandeirados. No entanto, o sistema TARP amplifica a velocidade do vento em 180\% (WEISBRICH; RAINEY 1977), que pode gerar problema estrutural significativo se ocorrer vento maior do que $16 \mathrm{~m} \mathrm{~s}^{-1}$.

Para evitar tal hipótese deve ser lembrado que o sistema TARP atua segundo as leis de circulação sobre cilindros definida por Abbott e Von Doenhoff (1959) e é diretamente proporcional à velocidade do vento e ao raio do cilindro se a geometria "cilíndrica" 
Figura 8 - Projeções do sistema eólico, as vistas em perfil e planta não possuem o sistema TARP, notar a base móvel
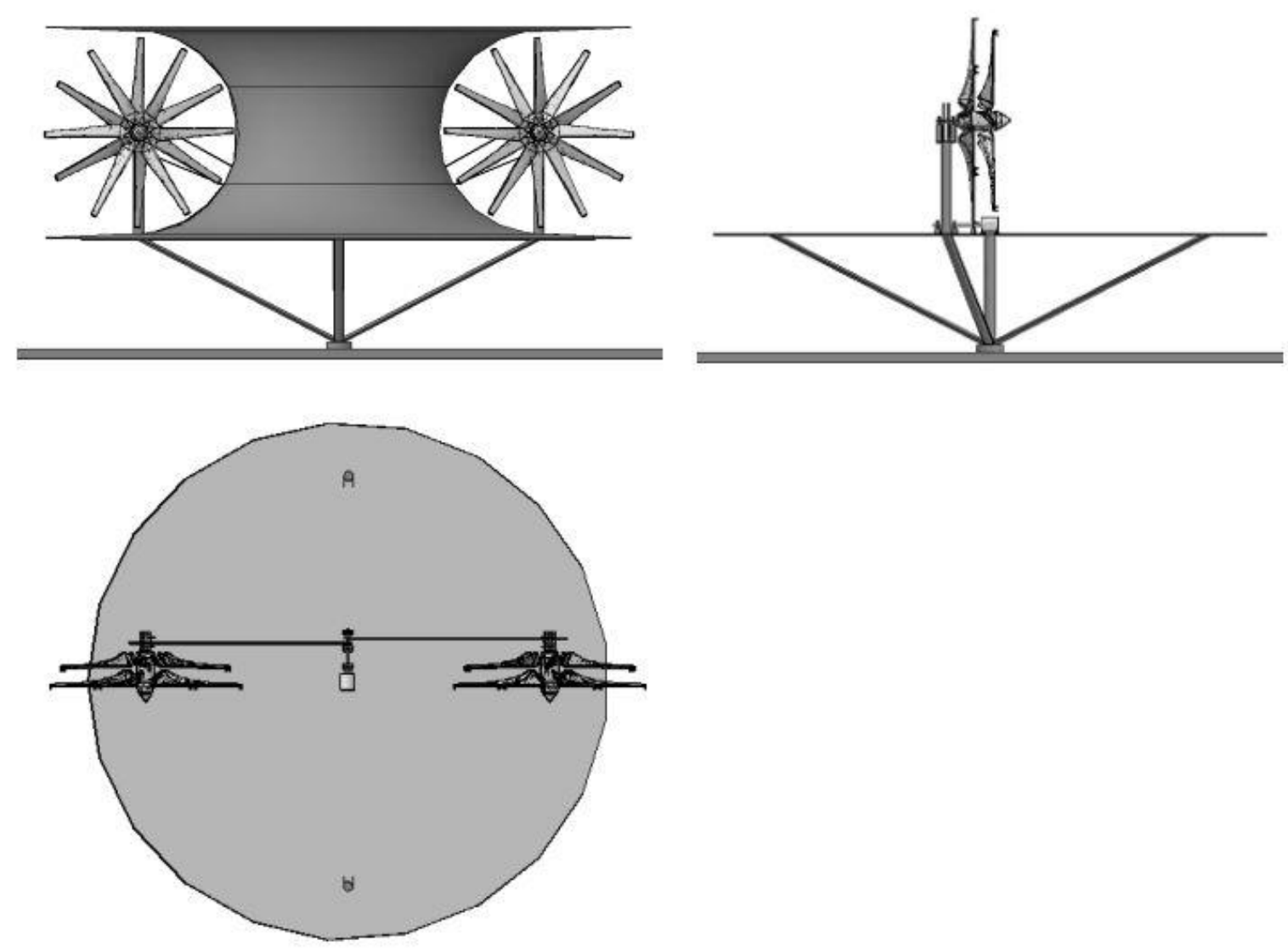

do Toro for modificada, o fenômeno de circulação é gravemente modificado diminuindo significativamente a amplificação da velocidade do vento. Para isto foi idealizado um sistema de venezianas que abririam em torno do tronco do toróide.

Em estimativa o peso de cada sistema contrarotativo com sua torre é aproximadamente $250 \mathrm{~N}$, o gerador pesa em torno de $100 \mathrm{~N}$, a base constituída de pranchas de madeira de $0,007 \mathrm{~m}$ e os tubos de aço da base móvel com diâmetro de $0,1 \mathrm{~m}$ tem peso em torno de 700 N. Portanto o peso total com o sistema TARP constituído por fibras de carnaúba de revestimento e bambu como estrutura é de $1.300 \mathrm{~N}$. Uma estimativa aproximada de preço é dada na Tabela 2.

A estimativa do custo total deste sistema eólico de geração de energia elétrica é significativamente menor em relação a sistemas comerciais importados atuais, na categoria de $1 \mathrm{~kW}$. No entanto, uma grande vantagem em comparação a operacionalidade deste sistema com os importados é que todos os componentes utilizados são de fácil obtenção em qualquer cidade pequena do Brasil.
Tabela 2 - Estimativa de custo do sistema eólico proposto

\begin{tabular}{lr}
\hline \multicolumn{1}{c}{ Elemento } & Valor $(\mathrm{R} \$)$ \\
\hline Pás de fibra de carnaúba e resina de mamona & $2.500,00$ \\
Motor de indução Trifásico blindado-gerador & 900,00 \\
Sistemas contra-rotativos & $2.000,00$ \\
Sistemas de controle de passo & $1.500,00$ \\
Sistema de transmissão de potência & $1.500,00$ \\
Prancharia de madeira, bambu e fibras & $1.500,00$ \\
Tubos de aço (2” x 5 mm) & $1.500,00$ \\
TOTAL & $11.400,00$ \\
\hline
\end{tabular}

Martins, Guarnieri e Pereira (2008) declaram que o rápido desenvolvimento da tecnologia e do mercado tem grandes implicações sócio-econômicas, e a energia eólica não foge a regra. Melício e Mendes (2007) e Sako (2009) reforçam escrevendo que uma das alternativas para diminuir o impacto poluente das atuais fontes de energia é a energia eólica. 
Daher (1997) deixa claro o enorme desafio que é operar sistemas de geração eólica baseados em geradores de imãs permanentes, tanto em termos de custos de aquisição como de manutenção para comunidades de poucos recursos e distantes dos grandes centros. A utilização de um motor de indução trifásico é excelente sugestão de Daher (1997), que embora não tenha eficiência grande, tem custo muito inferior aos sistemas geradores utilizados nas turbinas importadas, além da manutenção ser extremamente facilitada em território nacional.

Jara (2009) afirma que para haver produção plena de energia através do vento são necessários ventos de $12 \mathrm{~m} \mathrm{~s}^{-1}$. Isto representa, segundo Huang e Tsai (2004), baixos números de Reynolds que tem pequenos efeitos de blocagem, portanto significa maior eficiência aerodinâmica. Ross e Altmann (2011) explicam que a blocagem é criada pela redução na área da seção por onde o fluxo passa em comparação ou fluxo sem turbulência. Nakano et al. (2007) provaram que em fluxo laminar (baixos números de Reynolds) a separação e reagrupamento da camada limite sobre um aerofólio se dá próximo ao bordo de fuga para pequenos ângulos de ataque. Isto define a orientação do rotor segundo Sicot et al. (2008). Neste contexto a turbina projetada supera estes problemas e com a tecnologia de winglets o panorama fica ainda melhor.

Guerrero et al. (2012) e Lee, Kim e Kim (2012) destacam que winglets diminuem muito o arrasto e George (2011) afirma que o arrasto induzido é responsável por $40 \%$ do arrasto total de um aerofólio e Jones e Lasinski (1980) afirmam que winglets diminuem em $15 \%$ o arrasto induzido. Já em relação à sustentação Meyer e Covell (1986) afirmam que os winglets aumentam em $20 \%$. Hossain (2011) explica que os winglets atuam difundindo os fortes vórtices da ponta de asa melhorando a distribuição de sustentação na asa.
Outro ponto de destaque é que o sistema proposto pretende ser operado continuamente sem utilização de sistemas de armazenamento de energia, tais como baterias. Esta consideração é possível pela utilização do sistema TARP, que gera a potência de projeto a velocidades deveras baixas $\left(3,5 \mathrm{~m} \mathrm{~s}^{-1}\right)$. Pela avaliação do Atlas do Potêncial Eólico Brasileiro (CENTRO DE REFERÊNCIA PARA ENERGIA SOLAR E EÓLICA, 2001) pode-se perceber que em toda a área onde a agricultura familiar se desenvolve no Estado do Ceará, existe alta probabilidade de ventos constantes durante o ano nesta categoria de vento.

Uma relativa complexidade do sistema se forma na necessidade da utilização de dois sistemas de rotores contra-rotativos (Figura 9). Esta solução foi aventada pela necessidade de atendimento da requisição de potência elétrica definida no início do projeto $(1 \mathrm{~kW}$ de potência útil). No entanto, para aplicações de menor potência, todo o sistema poderia ser simplificado sendo constituído de apenas um conjunto contra-rotativo.

Atualmente o estado da arte em distribuição de energia elétrica se baseia no conceito de geração de distribuição (PORTOLANN; CAMPAGNOLO, 2010) e redes inteligentes (PENG; YAN, 2011). Estas configurações devem, segundo Garcia et al. (2012), ter pouca sensibilidade em relação a variações nos parâmetros de geração tais como voltagem e amperagem.

A geração distribuída possibilita a pulverização das fontes geradoras, diminuindo custos e aumentando a eficiência, principalmente em regiões isoladas, enquanto as redes inteligentes possibilitam que um consumidor que tenha a capacidade de geração se ligue a rede e venda o excedente de sua produção para a concessionária. Segundo Ge et al. (2011) as redes inteligentes são a tendência atual para distribuição de

Figura 9 - Sistema de transmissão de potência dos rotores para o gerador (centro da base)
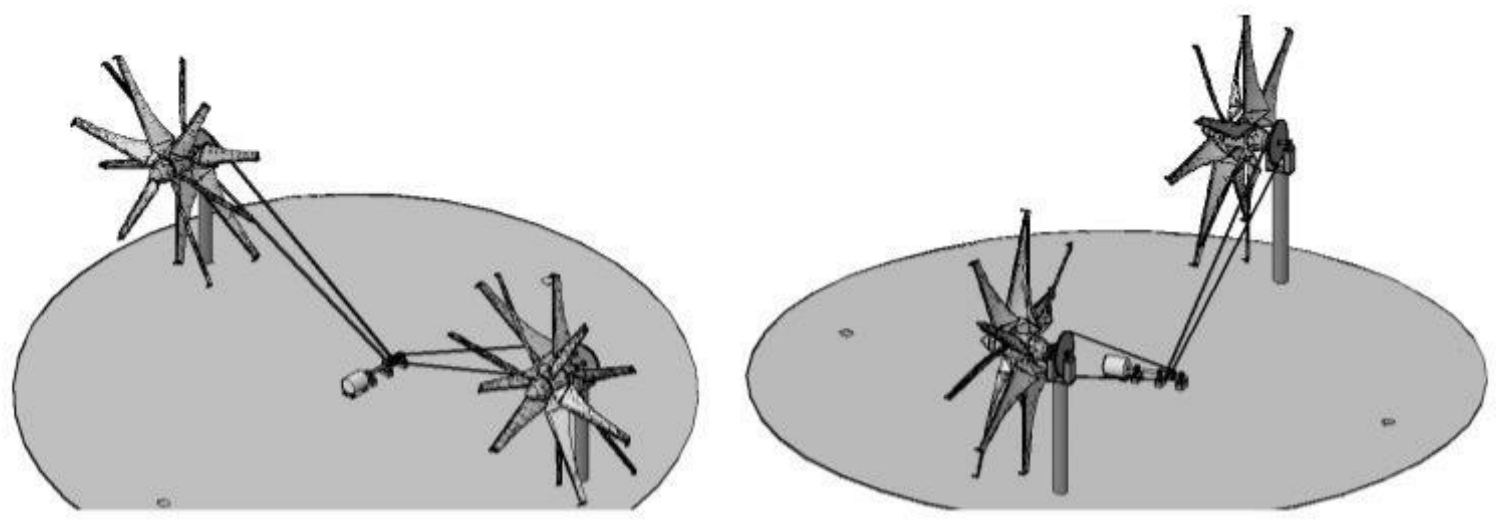
energia e caracterizam-se como inevitáveis no atual panorama elétrico mundial.

Neste contexto, percebe-se que o sistema proposto se adequa totalmente à realidade moderna, pois tecnicamente as turbinas eólicas têm grande potencial para aumentarem a eficiência global (USTUNTAS; SAHIN, 2008), que junto ao programa do Governo Federal, Luz para Todos (BRASIL, 2011), possibilitou a ligação da rede nacional de energia elétrica praticamente a todos os agricultores familiares do Nordeste Brasileiro, assim nos períodos em que a bomba de irrigação não estiver sendo utilizada, este excedente de energia elétrica pode ser vendido à concessionária. Este panorama é garantido pela Resolução Normativa da ANEEL REN-482 que estabelece as condições gerais para o acesso de microgeração e minigeração distribuída aos sistemas de distribuição de energia elétrica e o sistema de compensação de energia elétrica (AGÊNCIA NACIONAL DE ENERGIA ELÉTRICA, 2013).

A principal vantagem em relação à irrigação é a pouca distância entre o receptor de energia e o gerador (CARVALHO, 2003). Segundo Camacho e Ruedas (2011) este tipo de sistema é o mais efetivo e prático para geração. Por outro lado, nos períodos chuvosos, quando a velocidade do vento cai a praticamente zero, os agricultores terão assegurado sua irrigação, pois estão ligados à rede de energia elétrica e têm "créditos" com a concessionária.

\section{CONCLUSÃO}

O projeto de um sistema de geração elétrica através da energia eólica para agricultura familiar na região semiárida do Estado do Ceará foi concluído com sucesso, todos os sistemas foram conceituados e dimensionados, culminando em uma turbina eólica inovadora e adequada às necessidades a que se propôs atender. Pelas estimativas, tal sistema é capaz de fornecer energia a baixo custo e supostamente através de redes inteligentes e a geração distribuída poderá gerar renda.

\section{AGRADECIMENTOS}

Aos Professores Sérgio Daher, Paulo César Marques Carvalho e Paulo Alexandre Costa Rocha Ao Centro de Ciências Agrárias da Universidade Federal do Ceará pelo apoio e ao CNPq pela Bolsa de Produtividade em Desenvolvimento Tecnológico e Extensão Inovadora (DT).

\section{REFERÊNCIAS}

ABBOTT, I. H.; VON DOENHOFF, A. E. Teory of wing sections. New York: Dover, 1959. 693 p.

AIRFOIL INVESTIGATION DATABASE. Description of Airfoil E61. 2011. Disponível em: <http://www.w3.org>. Acesso em: 11 nov. 2011.

AGÊNCIA NACIONAL DE ENERGIA ELÉTRICA. Resolução Normativa REN-482. 2012. Disponível em: <http:// http://www. aneel.gov.br/cedoc/ren2012482.pdf. >. Acesso em: 03 abril de 2013.

ÁVILA, M. E. G. et al. O. Potencial de la energia eólica em zonas rurales de México. Interciências, v. 31, n. 4, p. 5-15, 2006.

BARIN, A. et al. Seleção de fontes alternativas de geração distribuída baseada em AHP e lógica Fuzzy. Revista SBA, v. 21, n. 5 , p. $120-130,2010$

BRASIL. Ministério das Minas e Energia. Programa luz para todos. 2011. Disponível em: <http://www.luzparatodos.mme. gov.br.>. Acesso em: 13 nov. 2011.

BYSTRYK, J.; SULLIVAN, P. E. Small wind turbine control in intermittent wing gusts. J. Wind Eng. Ind. Aerodynomics, v. 99, p. 624-637, 2011.

CAMACHO, A. C.; RUEDAS, B. F. Incorparación de un modelo de generador eólico al análises de flujos. Ingenieria Investigación y Tecnologia, v. 12, n. 3, p. 311-320, 2011.

CARMICHAEL, B. H. Low Reynolds number airfoil survey. Hampton: NASA, 1982. 105 p.

CLARK, H. Counter rotating wind turbine system. San Francisco: California Energy Commission, 2003. 11 p.

COLLINS, J. A. Projeto mecânico de elementos de máquinas. Rio de Janeiro: LTC, 2006.

CARVALHO, P. Wind energy and greenhouse gases emission market: the Brazilian potential. Wind Engineering, v. 27, n. 2, p. 245-255, 2003.

CENTRO DE REFERÊNCIA PARA ENERGIA SOLAR E EÓLICA Atlas do potencial eólico brasileiro. Brasília: CRESESB, 2001. 50 p.

DAHER, S. Um sistema baseado em gerador de indução trifásico para aproveitamento da energia eólica. 1997. Dissertação (Mestrado em Engenharia Elétrica) - Universidade Federal do Ceará, Fortaleza, 1997.

DICORATO, M. et al. Wind farm stability analysis in the presence of variable-speed generators. Energy, v. 39, n. 1, p. 40-47, 2012.

ECK, B. Fans. Oxfort: Pergamon Press, 1973.

FREIRE, W. J.; BERALDO, A. L. Tecnologias e materiais alternativos de construção. Campinas: Editora da UNICAMP, 2003.

FITZGERALD, A. E. et al. Máquinas Elétricas. Porto Alegre: Bookman, 2006.

GARCIA, J. L. D. et al. Indirect vector control of a squirrel cage induction generator wind turbine. Computer and Mathematics with applications, v. 63, n. 8, p.1310-1320, 2012. 
GE, Q. S. et al. Review of smart grid comprehensive assessment systems. Energy Procedia, v. 12, p. 219-229, 2011.

GEORGE, F. Understanding winglets technology. Disponível em: <http://printfu.org>. Acesso em: 12 nov. 2011.

GONZALEZ, F. D. et al. A review of energy storage technologies for wind power applications. Renewable and sustentaible energy reviews, v. 16, n. 4, p. 2154-2171, 2012.

GUERRERO, J. E. et al. Biomimetic spiroid winglets for lift and drag control. Comptes Rendus Mecanique, v. 340, n. 1/2, p. $67-80,2012$

HABASH, R. W. Y. et al. Performance of acontrarotating small wind energy converter. International Scholarly Research Network, 2011. Disponível em: <http://www.hindawi.com/isrn/mechanical. engineering/2011/828739/>. Acesso em: 14 set. 2012.

HANSEN, M. H. Aeroelastic stability analysis of wind turbines using an eigenvalue approach. Wind Energy, v. 7, p. 133-143, 2004.

HOSSAIN, A. et al. Prediction of aerodynamics characteristics of an aircraft model with and without winglets. Aerospace science and technology, v. 15, n. 8, p. 595-605, 2011.

HUANG, R. F.; TSAI, F. C. Flow and mixing characteristics of swirling wakes in blockage effect regime. Journal of Wind Engineering, v. 92, n. 2, p. 199-214, 2004.

JARA, F. A. V. Foundations for offshore wind turbines. Revista ingenieria de construction, v. 24, n. 1, p. 33-48, 2009.

JOHANSEN, J.; SORENSEN, N. N. Numerical analysis of winglets on wind turbine blades using CFD. In: European Wind Energy Congress, 2007, Milano. Proceedings...Milano: EWEA, 2007. 1 CD-ROM.

JONES, R. T.; LASINSKI, T. A. Effect of winglets ond the induced drag of ideal wing shapes. Hampton: NASA, $1980.30 \mathrm{p}$.

LEE, S. W.; KIM, S. U.; KIM, K. H. Aerodynamic performance of winglets on the tip gap inlet in a turbine cascade. International J. of Heat and Fluid Flow, v. 33, p. 145-157, 2012.

MARIA, P. H. S. et al. Modelagem numérica em alta resolução para previsão de geração de energia eólica no Ceará. Revista Brasileira de Metereologia, v. 23, n. 4, p. 477-489, 2008.

MARTINS, F. R. GUARNIERI, R. A.; PEREIRA, E. B. O aproveitamento da energia eólica. Revista Brasileira de Ensino de Física, v. 30, n. 1, 1304-1317, 2008.

MANWELL, J. F.; MCGOWAN, J. G.; ROGERS, A. L. Wing energy explained. West Sussex: Wiley, 2009. 705 p.

MELÍCIO, R.; MENDES, V. M. F. Simulacion de convertidores de potencia em sistemas eólicos. Informacion Tecnológica, v. 18, n. 4, p. 25-34, 2007.

MEYER, R. R.; COVELL, P. F. Effects of winglets on first generation jet transport wing. Hampton: NASA, 1986. 59 p.

NAKANO, T. et al. Experimental study on flow and noise characteristics of NACA0018 airfoil. Journal of Wind Enginnering, v. 95, n. 7, p. 511-531, 2007.
NHO, Y. C. et al. Effect of turbine blade tip shape on total pressure loss and secondary flow. International J. of Heat and Fluid Flow, v. 33, n. 1, p. 92-100, 2012.

PARK, J. The wind power book. Palo Alto: Cheshire Book, 1981. $246 \mathrm{p}$.

PEREIRA, L. A. A. et al. Wake and aerodynamics loads in multiple bodies-application to turbomachinery. Journal of Wind Engineering, v. 92, p. 477-491, 2004.

PENG, L.; YAN, G. S. Clean energy grid - connected technology based on smart grid. Energy Procedia, v. 12, p. 213-218, 2011.

PINILLA, M; MARTINEZ, S. Optimal design of permanentmagnet direct drive generator for win energy. Renewable Energy, v. 41, p. 267-276, 2012.

PORTOLANN, C. A.; CAMPAGNOLO, J. M. Controle de frequência híbrido em sistemas elétricos com incidêcia de geração distribuiída. SBA: Controle eAutomação, v. 21, n. 5, p. $250-260,2010$.

RAMIREZ, D. et al. Improvements in the grid connection of renewable generators with full power converters. Renewable Energy, v. 43, p. 90-100, 2012.

ROCHA, P. A. C. et al. Montagem e testes de uma bancada para medição de desempenho de aerogeradores em escala. Revista Brasileira de Ensino de Física, v. 32, n. 2, p. 23092314, 2010.

ROSS, I.; ALTMAN, A. Wind tunnel blockage corrections in savonius vertical axis wind turbine. Journal Wind Eng. Ind. Aerodynamics, v. 99, n. 5, p. 523-538, 2011.

SAKO, M. J. L. Apoyo público a la energia eólica en espana. Revista de Derecho, v. 31, p. 224-255, 2009.

SICOT, C. et al. Rotational and turbulence effects on a wind turbine blade. Journal Wind Eng. Ind. Aerodynamics, v. 96, n. 8/9, p. 1320-1331, 2008.

SORENSEN, J. D. et al. Effective turbulence models and fatigue reliability in wind farms. Prob. Eng. Mechanics, v. 23, n. 4, p. 531-538, 2008.

USTUNTAS, T.; SAHIN, A. D. Wind turbine power curve estimation. Journal of Wind Engineering, v. 96, n. 5, p. 611$620,2008$.

VOGT, H. H. Análise estrutural de pás de gerador eólico de pequeno porte feitas de fibra vegetal brasileira. 2010. Dissertação (Mestrado em Ciências Físicas Aplicadas) Universidade Estadual do Ceará, Fortaleza, 2010.

WANG, F. et al. The methodology for aerodynamic study on small domestic wind turbine with scoop. Journal of Wind Engineering, v. 96, n. 1, p. 1-24, 2008.

WEISBRICH, W. A.; RAINEY, D. Toroidal accelerator rotor platform (TARP). US 4156579 A, 12 nov. 1974, 25 fev. 1977

WHITCOMB, R. T. A design approach and selection wind tunnel results at high subsonic speed for wing tips mounted Winglets. Hampton: NASA, 1976. 60 p. 